\title{
Review of: "Screw stripping and its prevention in the hexagonal socket of 3.5-mm titanium locking screws"
}

\section{Eran Keltz}

Potential competing interests: The author(s) declared that no potential competing interests exist.

This study engages a real life problem in a systematic approach. However, some choices of the design are cumbersome and requires clarifications or corrections.

Title

I'm not sure whether stripping is the exact word for this problem. Although it had been used in the past by some authors, to me stripping is the distortion of the screw's thread to bone interface, and not the wear of the screw's head socket. Webster dictionary defines "strip" as "to tear or damage the thread of (a separable part or fitting)".

\section{Materials and Methods}

Preparation of jammed locking screws - The two last sentences are not clear. There is a mix of results in the methods section. If the intention of the authors that a pilot group was designed to decide the required torque for jamming it should be made clear in the text.

However, a torque level which is triple than the manufactures orders seems quite odd to me, and may yield in a mismatch between the results and clinical real life.

It is not clear how many groups are, and in which ones was the non dominant hand used. It was only made clear to me when reading the results. I would suggest a better description for this issue.

Why hadn't the introduction of interposed material tried when using the the T handle, the wrench or the non dominant hand with the straight driver? Is there any philosophy behind this choice? Has the study been tested by a single surgeon? Multiple? Were they skilled surgeons, trainees or not experienced at all? Please specify and discuss accordingly.

\section{Results}

Poorly described. $2^{\text {nd }}$ paragraph has its first sentence in bold with two dots. Unclear what it means. Odds ratio is used to describe the probability of an effect to occur, rather than not occur (in this case - the wear of the screw's socket). I would suggest to alter the numerator and the denominator in its calculation. The ratio of removed and jammed screws (so called, by mistake to my taste - "stripped") is staggering in all groups. It is of no comparison to real clinical life. This should challenge the selected jamming torque to start with, and should be discussed. 


\section{Discussion}

$1^{\text {st }}$ paragraph gives a thorough basic sense to the phenomenon.

Other than discussing the option of sharp edged and relatively large caliber screwdriver, the re is no discussion (!).

The discussion does not address the even better results of the hex key wrench. It's effect due to a short deforming lever arm is suggested in figure 5 but not discussed.

It does not address the non dominant hand as well, although its massive failure rate.

It does not address the interposition method although it is widely and successfully used.

\section{Limitations}

the sentence "The results may be different, with different torques applied to overtighten the screw" is a bit vague. I guess the authors mean that in real life overtightening occurs in variable torques. That's obvious. Again, it's unclear why the tripled amount of torque was selected.

These results should be discussed in the perspective of the testing surgeon/surgeons. Again - the failure rate is staggering.

Minor remarks:

$1^{\text {st }}$ paragraph "and lower risk of infection and are isoelastic" - consider rephrasing.

\section{Summary:}

This manuscript should be revised thoroughly, and only then considered for publication 